\title{
Theoretical Development of the Information Preservation Method for Strongly Nonequilibrium Gas Flows
}

\author{
Quanhua Sun ${ }^{*}$ and Iain D. Boyd ${ }^{\dagger}$ \\ University of Michigan, Ann Arbor, MI 48109
}

\begin{abstract}
The information preservation method is a particle technique for nonequilibrium gas flows with low statistical fluctuations. The foundation of the information preservation method is derived theoretically in this study. First, the evolution of the average information is obtained using Maxwell's equation of change. Then, the update of individual particle information is assumed to follow the governing equation of the average information. Two approaches are proposed to evaluate the statistical terms appeared in the governing equations. Namely, the local thermodynamic equilibrium approach and the flux splitting approach. It is found that the flux splitting approach is better, and can predict the shock structure of normal shock waves and the temperature distribution of thermal Couette flows for all Knudsen numbers.
\end{abstract}

\section{Nomenclature}

c $\quad=$ particle's microscopic velocity

$f \quad=$ velocity distribution function

$k=$ Boltzmann constant

$\mathrm{Kn} \quad=$ Knudsen number

$m \quad=$ molecular mass

$\mathrm{Ma} \quad=$ Mach number

$n=$ number density

$p \quad=$ pressure

$Q \quad=$ particle property

$R \quad=$ gas constant

$T=$ temperature

$t \quad=$ time

$\mathbf{V}=$ particle's preserved velocity

$\rho \quad=$ mass density

',",'" = superscripts indicating velocity fluctuations

$0=$ subscript indicating mean velocity

c $=$ subscript indicating cell property

\section{Introduction}

$\mathrm{T}$ HE direct simulation Monte Carlo (DSMC) method ${ }^{1}$ is a widely used particle approach for simulating rarefied gas flows. It has been very successful for solving problems in the field of high-speed rarefied gas flows. Its recent applications in micro-scale gas flows, however, have shown several challenges for simulating low-speed flows. ${ }^{2}$ The major challenge comes from the difficulty to reduce the statistical scatter to an acceptable level. Unlike for high-speed flows, it requires a huge sample size for a DSMC simulation of a low-speed gas flow to obtain a meaningful noise-to-signal ratio. This, of course, makes the DSMC method so numerically expensive to be unacceptable for many low-speed flow simulations.

Many efforts have been made to reduce the statistical scatter associated with the DSMC method, including a post-processing technique ${ }^{3}$ and several modified DSMC approaches ${ }^{4,5}$. One successful approach is the development of the information preservation (IP) method. ${ }^{6,7}$ The IP method was first proposed by Fan and Shen to simulate uni-

\footnotetext{
* Research Fellow, Department of Aerospace Engineering, 1320 Beal Avenue, Senior Member AIAA.

${ }^{\dagger}$ Professor, Department of Aerospace Engineering, 1320 Beal Avenue, Associate Fellow AIAA.
} 
directional, constant-density, low-speed rarefied gas flows. ${ }^{8}$ They proposed to preserve a so-called "information velocity" in particles simulated in the DSMC simulation. The information velocity was set at the macroscopic level, which was initialized when a particle was introduced into the simulation; and the flow velocity was sampled from the information velocity of particles instead of the microscopic velocity. It was shown that this sampling exhibited much less statistical scatter than regular DSMC sampling for low-speed flows, and thus an IP simulation can save computational time by several orders of magnitude compared with a similar DSMC simulation. Therefore, it is not surprising that many developments have been made to extend the IP method for more general flows. For instance, Cai et al. ${ }^{9}$ extended the IP method by preserving information velocity in two components for 2D isothermal compressible flows. A pressure force term was introduced to update the preserved information, and the flow density was solved using the continuum equation. Later, Sun and Boyd ${ }^{10}$ extended the IP method for general low-speed gas flow simulations by additionally preserving temperature information in particles. The validity of the IP method has been shown in many examples ranging from near-continuum flow to free molecular flow. ${ }^{6,9-14}$

The general procedure of a state-of-the-art IP method consists of four steps based on the standard DSMC method. First, simulated particles are assigned relevant flow information at the macroscopic level as preserved information when the particles are introduced into the simulation domain (either through initialization or from the flow boundaries). Second, particles move to new locations in a time step according to their microscopic velocity. Third, whenever a particle collision occurs, two particles adjust their preserved information with a requirement of conserving the total momentum and energy. Fourth and finally, every particle has its preserved information updated to include effects excluded during the movement and collision processes. This general procedure is based on the physical understanding of gas flows, and is not derived theoretically. Many versions of the IP method ${ }^{6,9-11,15}$ have been reported where good results were obtained for specific problems. Therefore, it is very important to understand the theoretical aspects of the IP method.

In this paper, we intend to explain the theoretical foundation of the IP method. First, general expressions are derived in the next section using Maxwell's equation of change. Second, detailed expressions are evaluated under several approximations. Then, the schemes are tested using two different nonequilibrium flow examples. Finally, the paper ends with some concluding remarks.

\section{Foundation of the Information Preservation Method}

It is known that the DSMC method is equivalent to solving the Boltzmann equation for rarefied gas flows. ${ }^{1}$ Particles in a DSMC simulation represent a discrete form of the velocity distribution function. Thus the flow fields can be obtained by sampling the particle information. In the IP method, the particles not only have the microscopic velocity, but also preserve the information velocity. The preserved information is sampled to obtain the flow information. Hence, the evolution of the preserved information is critical to the accuracy of IP simulations.

The preserved information is carried by each particle. However, it is unclear what equation governs the preserved information of each particle. On the other hand, Maxwell's equation of change is a governing equation for the average of general properties associated with particles. Hence, we start from Maxwell's equation of change to derive equations for the preserved information. The conservation form of Maxwell's equation of change can be written as follows: ${ }^{1}$

$$
\frac{\partial}{\partial t}(n \bar{Q})+\nabla \cdot(n \overline{\mathbf{c}} Q)=\Delta[Q]
$$

where the collision integral $\Delta[Q]$ is a weighted average of the change in the total value of particle property $Q$ before and after collisions, and the external force term is neglected. For the sake of simplicity, only monatomic atoms are considered in this study. Several notations are explained as follows for clarity: the microscopic velocity of a particle is denoted by $\mathbf{c}$, the preserved velocity is expressed as $\mathbf{V}$, whereas the macroscopic velocity of the flow field is written as $\mathbf{c}_{0}$. These velocities are connected through velocity fluctuations: $\mathbf{c}^{\prime}=\mathbf{c}-\mathbf{c}_{0}, \mathbf{c}^{\prime \prime}=\mathbf{V}-\mathbf{c}_{0}$, and $\mathbf{c}^{\prime \prime \prime}=\mathbf{c}-\mathbf{V}$, whereas the average of each fluctuation is zero because the total momentum must be the same whether it is in preserved form or in microscopic form.

\section{A. Average of Preserved Velocity}

If quantity $Q$ in the equation of change is set as momentum $m \mathbf{c}$, then the equation of change reads as:

$$
\frac{\partial}{\partial t}(n m \overline{\mathbf{c}})+\nabla \cdot\left(n m \overline{\mathbf{c}^{2}}\right)=\Delta[m \mathbf{c}]=0
$$

since total momentum is conserved during a collision. With the help of the fluctuations, it can be easily shown that $\overline{\mathbf{c}}=\overline{\mathbf{V}}$ and $\overline{\mathbf{c}^{2}}=\overline{\mathbf{c V}}+\overline{\mathbf{c}^{\prime} \mathbf{c}^{\prime \prime \prime}}$. Substituting these expressions into Eq. (2), we obtain the following momentum equation 


$$
\frac{\partial}{\partial t}(n m \overline{\mathbf{V}})+\nabla \cdot(n m \overline{\mathbf{c}})=-\nabla \cdot\left(n m \overline{\mathbf{c}^{\prime \prime} \mathbf{c}^{\prime}}\right)+\Delta[m \mathbf{c}]
$$

Equation (3) is the governing equation for the average of the preserved velocity. The first term of the left hand side is the change rate of the momentum. The second term is the change due to the microscopic movement of the particles, where the preserved velocity is carried by the particles. The first term of the right hand side is a microscopic correlation term, which relates to the average of the product of two fluctuations. The final term is the collision integral term. It is kept in the equation although its value is zero to indicate the existence of particle collisions.

\section{B. Average of Preserved Temperature}

If quantity $Q$ in the equation of change is set as translational energy $\frac{1}{2} m c^{2}$, then the equation of change reads as:

$$
\frac{\partial}{\partial t}\left(\frac{1}{2} n m \overline{c^{2}}\right)+\nabla \cdot\left(\frac{1}{2} n m \overline{\mathbf{c} c^{2}}\right)=\Delta\left[\frac{1}{2} m c^{2}\right]=0
$$

The energy can also be expressed in the preserved form as $\frac{1}{2} m\left(V^{2}+3 R T\right)$. Then we have a relation $\overline{c^{2}}=\overline{V^{2}+3 R T}$ to ensure the average energy be the same in both microscopic and preserved forms. Furthermore, it can be shown that $\overline{\mathbf{c} c^{2}}=\overline{\mathbf{c}\left(V^{2}+3 R T\right)}-\overline{\mathbf{c}^{\prime}\left(V^{2}+3 R T-c^{2}\right)}$. Substituting these two expressions into Eq. (4), we obtain the following energy equation:

$$
\frac{\partial}{\partial t}\left(\frac{1}{2} n m \overline{\left(V^{2}+3 R T\right)}\right)+\nabla \cdot\left(\frac{1}{2} n m \overline{\mathbf{c}\left(V^{2}+3 R T\right)}\right)=\nabla \cdot\left(\frac{1}{2} n m \overline{\mathbf{c}^{\prime}\left(V^{2}+3 R T-c^{2}\right)}\right)+\Delta\left[\frac{1}{2} m c^{2}\right]
$$

which is the governing equation for the average of the preserved temperature (or energy). The meaning of each term in Eq. (5) is similar to the corresponding term in Eq. (3).

\section{Individual Preserved Information}

Equations (3) and (5) govern the evolution of the average of the preserved velocity and temperature, respectively. These equations are used to derive evolution equations for the preserved information of individual particles. If $\mathbf{V}=\mathbf{c}$ and $V^{2}+3 R T=c^{2}$, then Eqs. (3) and (5) are actually the governing equations for the microscopic information. The evolution of this specific individual preserved information follows the procedure in the standard DSMC method, and can be connected with the governing equations for the average of the preserved information. Namely, the information follows the particle movement (the movement term) and is updated during collisions (collision term). The correlation terms can also be included since they are zero in this case. It is then assumed that a similar connection applies to a general case of the preserved information for the evolution equations. In addition, it is assumed that the correlation terms apply equally to individual particles. With these two assumptions, the governing equations for individual preserved information are expressed as follows:

$$
\begin{gathered}
\frac{\partial}{\partial t}(n m \mathbf{V})+\text { movement }=-\nabla \cdot\left(n m \overline{\mathbf{c}^{\prime \prime \prime} \mathbf{c}^{\prime}}\right)+\text { collision } \\
\frac{\partial}{\partial t}\left(\frac{1}{2} n m\left(V^{2}+3 R T\right)\right)+\text { movement }=\nabla \cdot\left(\frac{1}{2} n m \overline{\mathbf{c}^{\prime}\left(V^{2}+3 R T-c^{2}\right)}\right)+\text { collision }
\end{gathered}
$$

\section{Flow Field}

The flow information in the IP method is obtained by sampling preserved information of particles in each cell of a computational mesh. The flow velocity is,

and the flow temperature is

$$
\mathbf{V}_{c}=\overline{\mathbf{c}}=\overline{\mathbf{V}}
$$

$$
T_{c}=\frac{1}{3 R} \overline{c^{\prime 2}}=\frac{1}{3 R}\left(\overline{c^{2}}-c_{0}^{2}\right)=\frac{1}{3 R}\left(\overline{V^{2}+3 R T}-c_{0}^{2}\right)=\bar{T}+\frac{1}{3 R}\left(\overline{V^{2}}-\bar{V}^{2}\right)
$$

The flow number density $n$, however, has to be calculated using the continuity equation:

$$
\frac{\partial}{\partial t}(n m)+\nabla \cdot\left(n m \mathbf{V}_{c}\right)=0
$$




\section{E. Summary of the IP Procedure}

The information preservation method is implemented on top of the DSMC method by preserving flow information in simulated particles. The detailed procedure is summarized as follows:

1) Every particle is given a velocity $\mathbf{V}$ and temperature $T$ when it is introduced into the simulation domain. The number density $n$ of the flow is initialized at the beginning of the simulation.

2) Particles move according to their microscopic velocity, which corresponds to the movement term in Eqs. (6) and (7).

3) Particles perform collisions where the collision pairs are determined by the DSMC collision scheme. The collision model for the preserved information requires further study, but clearly both momentum and energy must be conserved. This step corresponds to the collision term.

4) Preserved information in particles is updated following the governing equations, which read as follows after particle motions and collisions:

$$
\begin{gathered}
\frac{\partial}{\partial t}(n m \mathbf{V})=-\nabla \cdot\left(n m \overline{\mathbf{c}^{\prime \prime} \mathbf{c}^{\prime}}\right) \\
\frac{\partial}{\partial t}\left(\frac{1}{2} n m\left(V^{2}+3 R T\right)\right)=\nabla \cdot\left(\frac{1}{2} n m \overline{\mathbf{c}^{\prime}\left(V^{2}+3 R T-c^{2}\right)}\right)
\end{gathered}
$$

5) Flow field information is evaluated using Eqs. (8)-(10).

In this procedure, a collision model is still needed to handle the preserved information during collisions. For the time being, the collision model of Sun and Boyd ${ }^{10}$ is employed. Then the last question left is the evaluation of the correlation terms, which is discussed in the next section.

\section{Evaluation of the Correlation Terms}

The correlation terms in Eqs. (11) and (12) are in the statistical form. Accurate evaluation of these terms requires knowledge of the velocity distribution function. However, the velocity distribution function is usually unknown during simulation, and assumptions have to be made for the evaluation. In this study, we will propose two approaches and discuss their differences.

\section{A. Local Thermodynamic Equilibrium Approach}

The local thermodynamic equilibrium (LTE) approach assumes that the velocity distribution function can be described using preserved particle information where every particle represents a Maxwellian distribution. The velocity distribution of the particle system is expressed as

$$
f_{L T E}=\overline{f_{L T E, i}}=\overline{\frac{1}{(2 \pi R T)^{3 / 2}} \exp \left(-\frac{|\mathbf{c}-\mathbf{V}|^{2}}{2 R T}\right)}
$$

where the bar indicates the average over all particles. With this velocity distribution function, expression $\overline{\mathbf{c}^{\prime \prime \prime} \mathbf{c}^{\prime}}$ is evaluated as follows:

$$
\left.\overline{\mathbf{c}^{\prime \prime} \mathbf{c}^{\prime}}\right|_{L T E}=\iiint(\mathbf{c}-\mathbf{V})\left(\mathbf{c}-\mathbf{c}_{0}\right) f_{L T E} d \mathbf{c}=\overline{R T} \mathbf{I}
$$

and expression $\overline{\mathbf{c}^{\prime}\left(V^{2}+3 R T-c^{2}\right)}$ is as follows:

$$
\left.\overline{\mathbf{c}^{\prime}\left(V^{2}+3 R T-c^{2}\right)}\right)_{L T E}=\iiint\left(\mathbf{c}-\mathbf{c}_{0}\right)\left(V^{2}+3 R T-c^{2}\right) f_{L T E} d \mathbf{c}=-2 R \overline{T \mathbf{V}}
$$

Substituting these expressions into Eqs. (11) or (12), we get the following equations for step 4:

$$
\begin{gathered}
\frac{\partial}{\partial t}(n m \mathbf{V})=-\nabla \bar{p} \\
\frac{\partial}{\partial t}\left(\frac{1}{2} n m\left(V^{2}+3 R T\right)\right)=-\nabla \cdot(\overline{p \mathbf{V}})
\end{gathered}
$$

where

$$
p=n k T
$$

It is clear that the correlation terms are similar to the pressure terms in the Navier-Stokes equations. However, the pressure is not evaluated from the flow field temperature (see Eq. (9)). In addition, viscous effects do not appear in the correlation terms. Nevertheless, viscous effects are included in this scheme of the IP method through particle movements and collisions. 


\section{B. Flux Splitting Approach}

The local thermodynamic equilibrium approach is a quasi-equilibrium approach for the correlation terms, which can be regarded as the equilibrium solution of the correlation terms. A more accurate evaluation of the correlation terms is to include certain nonequilibrium characteristics of the flow, such as the flux splitting (FS) approach as proposed here. The average $\overline{A_{k}}$ is calculated by splitting the velocity distribution function into two parts and limiting the integration over half velocity space to particles whose microscopic velocity is within that half velocity space:

$$
\overline{A_{k}}=\frac{\sum_{i} \delta\left(c_{i, k}>a_{k}\right) \iiint_{c_{c_{k}}>a_{k}} A_{k} f_{L T E, i} d \mathbf{c}}{\sum_{i} \delta\left(c_{i, k}>a_{k}\right)}+\frac{\sum_{i} \delta\left(c_{i, k} \leq a_{k}\right) \iiint_{c_{k} \leq a_{k}} A_{k} f_{L T E, i} d \mathbf{c}}{\sum_{i} \delta\left(c_{i, k} \leq a_{k}\right)}
$$

which can also be expressed as

$$
\overline{A_{k}}=\left.\overline{\iiint_{c_{k}>a_{k}} A_{k} f_{L T E, i} d \mathbf{c}}\right|_{c_{i, k}>a_{k}}+\left.\overline{\iiint_{c_{k} \leq a_{k}} A_{k} f_{L T E, i} d \mathbf{c}}\right|_{c_{i, k} \leq a_{k}}
$$

where the summation $i$ is over all particles in the cell and $a_{k}$ is the splitting value for the velocity component in the $k$ direction that is normal to the interface.

We calculate $\overline{\mathbf{c}^{\prime \prime \prime} \mathbf{c}^{\prime}}$ and $\overline{\mathbf{c}^{\prime}\left(V^{2}+3 R T-c^{2}\right)}$ in the index form as follows:

$$
\begin{aligned}
\left.\bar{c}_{k}^{\prime \prime \prime} c_{j}^{\prime}\right|_{F S}= & \frac{\sum_{i} \delta\left(c_{i, k}>a_{k}\right)\left(\sqrt{\frac{R T}{2 \pi}}\left(V_{j}-c_{0 j}\right) \exp \left(-\frac{\left(V_{k}-a_{k}\right)^{2}}{2 R T}\right)+\delta_{k j}\left(\frac{R T}{2}\left(1+\operatorname{erf}\left(\frac{V_{k}-a_{k}}{\sqrt{2 R T}}\right)\right)-\sqrt{\frac{R T}{2 \pi}}\left(V_{j}-a_{j}\right) \exp \left(-\frac{\left(V_{k}-a_{k}\right)^{2}}{2 R T}\right)\right)\right)}{\sum_{i} \delta\left(c_{i, k}>a_{k}\right)} \\
+ & \frac{\sum_{i} \delta\left(c_{i, k} \leq a_{k}\right)\left(-\sqrt{\frac{R T}{2 \pi}}\left(V_{j}-c_{0 j}\right) \exp \left(-\frac{\left(V_{k}-a_{k}\right)^{2}}{2 R T}\right)+\delta_{k j}\left(\frac{R T}{2}\left(1-\operatorname{erf}\left(\frac{V_{k}-a_{k}}{\sqrt{2 R T}}\right)\right)+\sqrt{\frac{R T}{2 \pi}}\left(V_{j}-a_{j}\right) \exp \left(-\frac{\left(V_{k}-a_{k}\right)^{2}}{2 R T}\right)\right)\right)}{\sum_{i} \delta\left(c_{i, k} \leq a_{k}\right)}
\end{aligned}
$$

and

$$
\begin{aligned}
\frac{\left.V^{2}+3 R T-c^{2}\right) c_{k}^{\prime}}{F S} & =\frac{\sum \delta\left(c_{i, k}>a_{k}\left(-R T V_{k}\left(1+\operatorname{erf}\left(\frac{V_{k}-a_{k}}{\sqrt{2 R T}}\right)\right)-\sqrt{\frac{R T}{2 \pi}}\left(R T-\left(V_{k}+a_{k}\right)\left(c_{0 k}-a_{k}\right)\right) \exp \left(-\frac{\left(V_{k}-a_{k}\right)^{2}}{2 R T}\right)\right)\right.}{\sum_{i} \delta\left(c_{i, k}>a_{k}\right)} \\
& +\frac{\sum \delta\left(c_{i, k} \leq a_{k}\right)\left(-R T V_{k}\left(1-\operatorname{erf}\left(\frac{V_{k}-a_{k}}{\sqrt{2 R T}}\right)\right)+\sqrt{\frac{R T}{2 \pi}}\left(R T-\left(V_{k}+a_{k}\right)\left(c_{0 k}-a_{k}\right)\right) \exp \left(-\frac{\left(V_{k}-a_{k}\right)^{2}}{2 R T}\right)\right)}{\sum_{i} \delta\left(c_{i, k} \leq a_{k}\right)}
\end{aligned}
$$

If $a_{k}=\overline{c_{k}}$, expressions (20) and (21) can be simplified as follows:

$$
\begin{aligned}
& \left.\overline{c_{k}^{\prime \prime \prime} c_{j}^{\prime}}\right|_{F S, j=k}=\left.\frac{1}{2} \overline{R T}\right|_{c_{i, k}>\overline{c_{k}}}+\left.\frac{1}{2} \overline{R T}\right|_{c_{i, k} \overline{c_{k}}}+\left.\overline{\frac{1}{2}} \overline{\operatorname{Rerf}\left(\frac{V_{k}-\overline{c_{k}}}{\sqrt{2 R T}}\right)}\right|_{c_{i, k}>\overline{c_{k}}}-\left.\frac{1}{2} \overline{R \operatorname{Terf}\left(\frac{V_{k}-\overline{c_{k}}}{\sqrt{2 R T}}\right)}\right|_{c_{i, k} \overline{c_{k}}}
\end{aligned}
$$

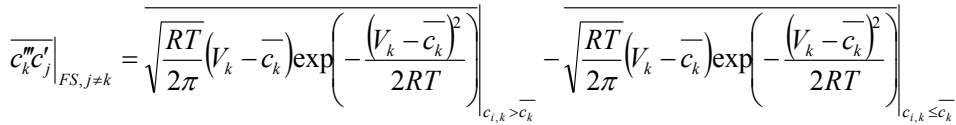

and

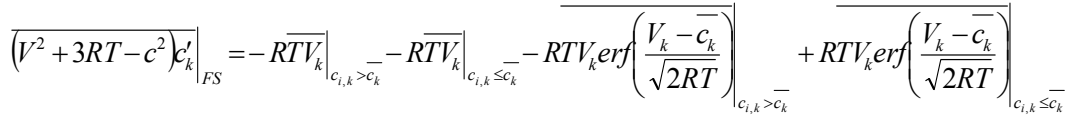

$$
\begin{aligned}
& -\left.\sqrt{\frac{R T}{2 \pi} R T \exp \left(-\frac{\left(V_{k}-\overline{c_{k}}\right)^{2}}{2 R T}\right)}\right|_{c_{i, k}>\overline{c_{k}}}+\left.\sqrt{\frac{R T}{2 \pi}} R T \exp \left(-\frac{\left(V_{k}-\overline{c_{k}}\right)^{2}}{2 R T}\right)\right|_{c_{i, k} \overline{c_{k}}}
\end{aligned}
$$

It is clear that the flux splitting approach predicts more terms for the correlation terms than the local thermodynamic equilibrium approach. Most of the terms, however, can be neglected if $\left(V_{k}-\overline{c_{k}}\right) / \sqrt{2 R T}$ is very small. An extreme case is the thermal-Couette flow where the flow velocity is zero. Then expression (22) reduces to 
expression (14). However, expression (23) still has more terms than expression (15) although it can be simplified as follows:

$$
\left.\overline{\left(V^{2}+3 R T-c^{2}\right) c_{k}^{\prime}}\right|_{F S}=-2 R \overline{T V_{k}}-\left.\sqrt{\frac{R T}{2 \pi}} R T\right|_{c_{i, k}>\overline{c_{k}}}+\left.\sqrt{\frac{R T}{2 \pi}} R T\right|_{c_{i, k} \leq \bar{c}_{k}}
$$

It is not surprising that these extra terms exist in the expression. From gas kinetic theory, the average translational energy carried by one particle across an interface is $2 k T$ whereas the corresponding volume average is only $3 k T / 2$. This difference of $k T / 2$ corresponds exactly to the extra terms in expression (24). Another approach to include this energy difference for the IP method is the additional energy flux approach ${ }^{10}$ in which the energy difference of $k T / 2$ is transferred when a particle crosses an interface. In this sense, the additional energy approach is a special case of the flux splitting approach.

\section{Numerical Examples}

The information preservation method has been applied to study many problems using several versions. For instance, excellent results have been obtained for Couette flow for a wide range of Knudsen number. ${ }^{10}$ The IP method predicts successfully the aerodynamics of a flat plate airfoil at very-low Reynolds numbers. ${ }^{14}$ However, the IP method also encounters difficulties when simulating high-speed flows. ${ }^{15}$ In this section, we intend to test the proposed schemes using the challenging examples that the IP method has encountered. The first example is the shock structure in argon gas, which is regarded as a benchmark problem for the DSMC method. The second example is the thermal Couette flow. This problem is used to test the consistency of the flux splitting approach and the additional energy flux model.

\section{A. Shock Structure in Argon}

Wang and Boyd $\mathrm{d}^{15}$ tested the IP method using 1-D stationary shock waves. They discovered that the additional energy flux model ${ }^{10}$ was not able to capture the shock structure, which is not surprising because that model was proposed for low-speed gas flows. Then they designed a new energy flux model by evaluating the energy flux in a way that is similar to the flux splitting approach. With this new energy flux model, a better shock structure was captured although they failed to exclude the particle movement when evaluating the energy flux. In this sub-section, we will evaluate the LTE and FS approaches using shock wave simulations by comparing to DSMC results and experimental data.

The flow conditions are the same as those in Wang and Boyd. ${ }^{15}$ Namely, the upstream conditions are: $T_{1}=300 \mathrm{~K}$ and $\rho_{1}=1.068 \times 10^{-4} \mathrm{~kg} / \mathrm{m}^{3}$. The downstream conditions are determined by the Rankine-Hugoniot relations. The computational domain is large enough to contain the entire shock wave. Particles are introduced into the computational domain through the inflow boundary and are initialized with the upstream conditions. Particles are carefully treated at the outflow boundary using moving piston boundary conditions. The preserved information of the incoming particles is assigned with the upstream values. When particles are reflected from the moving piston (outflow boundary), the downstream values are assigned as their preserved information. In order to stabilize the shock location, the total number control technique of Bird ${ }^{1}$ is also employed.

In a typical simulation, 400 cells are used to uniformly divide the computational domain in the flow direction whereas periodic boundary conditions are employed in the transverse direction. The cell size is about one tenth of the molecular mean free path at the upstream conditions. The time step is $10 \mathrm{~ns}$ for all cases considered. We use at least 100 particles in each cell to reduce the random walks.

The results for a weak shock wave having a Mach number of 1.2 are plotted in Figs. 1(a) and 1(b) for the LTE and FS approaches, respectively. The plotted non-dimensional variable $Q^{*}$ is defined as

$$
Q^{*}=\frac{Q-Q_{\text {ref }}}{\left|Q_{2}-Q_{1}\right|},
$$

where the subscripts 1 and 2 represent upstream and downstream conditions of the shock wave, respectively. $Q_{\text {ref }}$ is $\rho_{1}$ for density, $T_{1}$ for temperature, and $V_{2}$ for velocity. It is clear that the LTE approach fails to capture the shock structure whereas the FS approach predicts very good shock structure as compared with the DSMC result. The reason for the failure of the LTE approach is obvious because it omits many terms when the correlation terms are evaluated. Additional simulations for shock waves having different shock strength show that the LTE approach is unsuitable for shock wave simulations. We will then focus on the FS approach for further studies. 


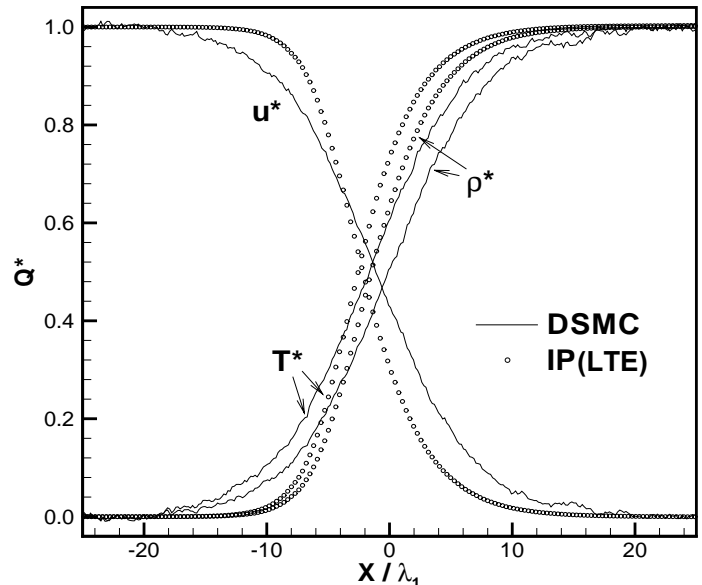

(a)

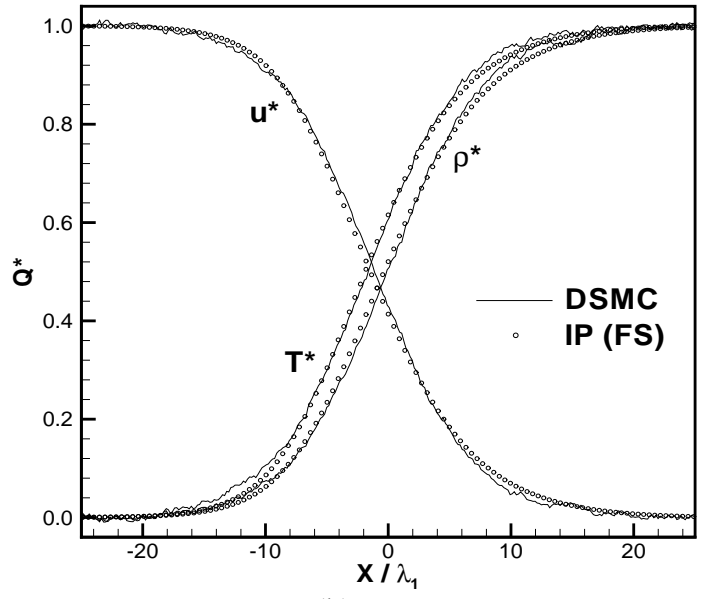

(b)

Figure 1. Comparison of shock structure for a Mach 1.2 shock wave. (a) the local thermodynamic equilibrium approach, (b) the flux splitting approach.

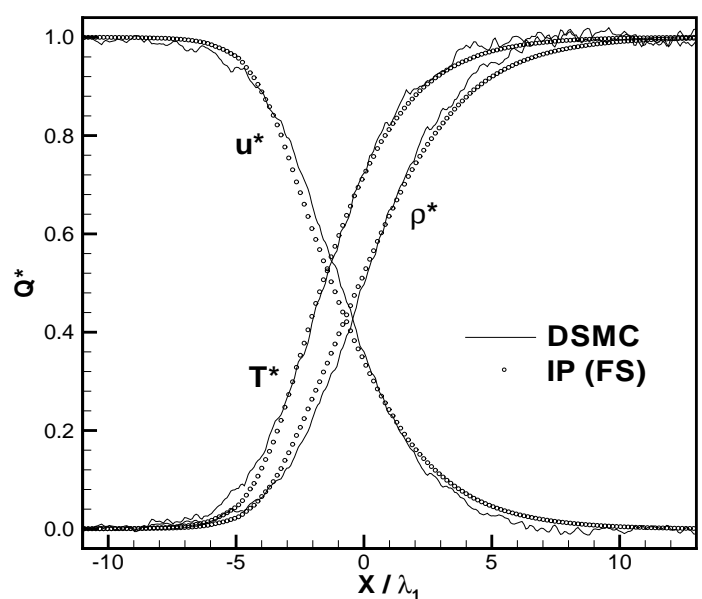

(a)

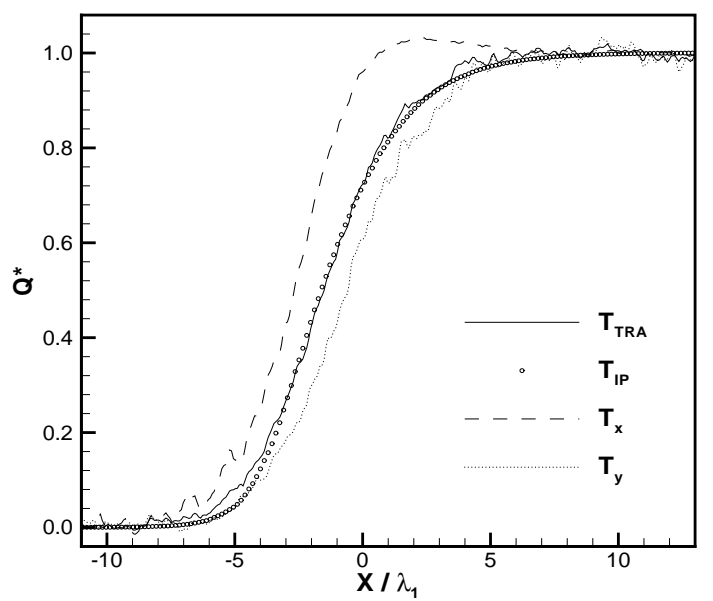

(b)

Figure 2. Shock structure for a Mach 1.55 shock wave. (a) non-dimensional flow properties, (b) nonequilibrium represented by temperatures.

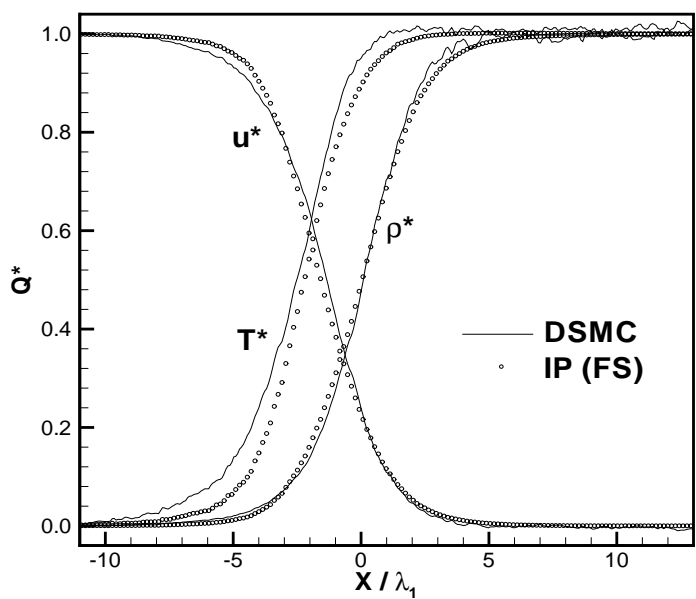

(a)

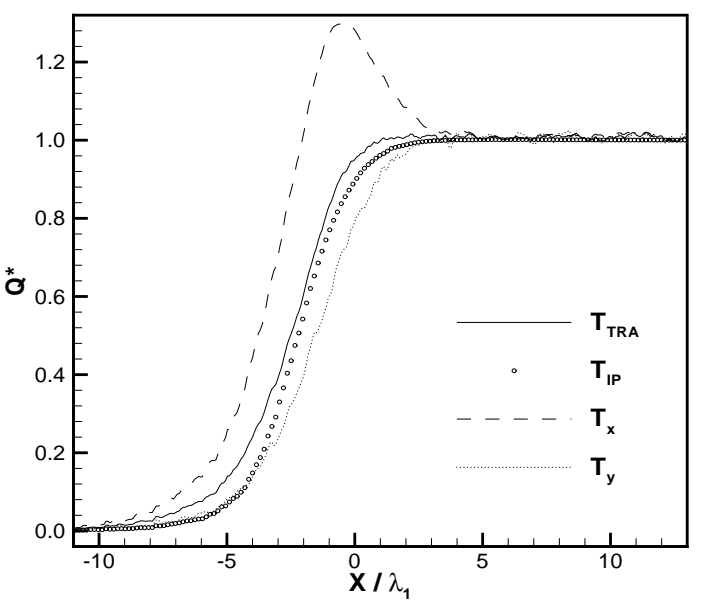

(b)

Figure 3. Shock structure for a Mach 5 shock wave. (a) non-dimensional flow properties, (b) nonequilibrium represented by temperatures. 


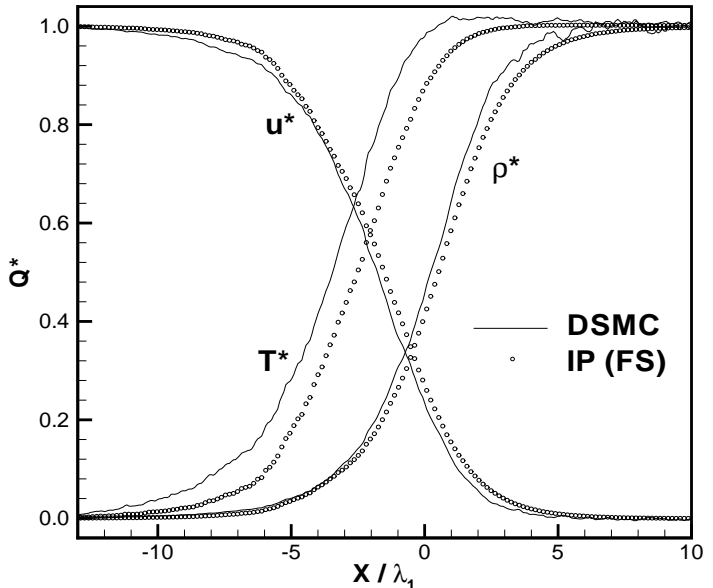

(a)

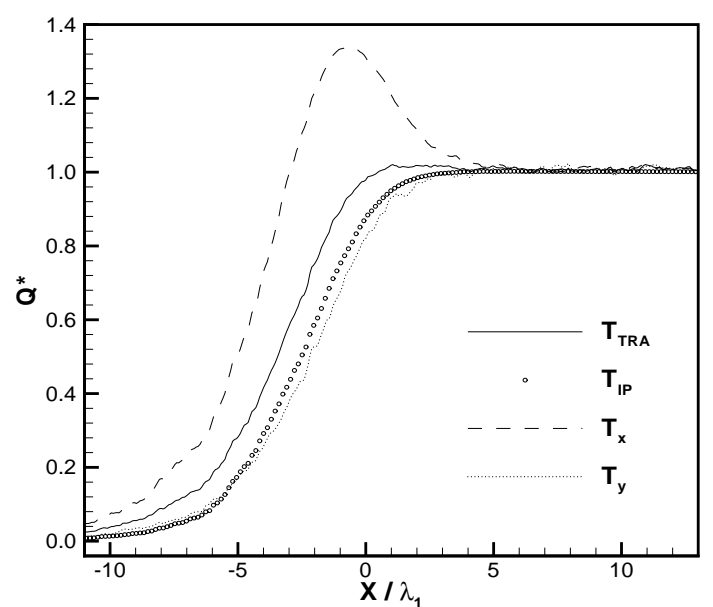

(b)

Figure 4. Shock structure for a Mach 8 shock wave. (a) non-dimensional flow properties, (b) nonequilibrium represented by temperatures.

When the shock strength of a shock wave increases, the flow becomes strongly nonequilibrium. It is therefore very interesting to examine the performance of the FS approach for shock structure of stronger shock waves. Results for three shock waves are plotted in Figs. (2)-(4), respectively. In general, the discrepancies of the shock structure between the IP and DSMC results slightly increase when the shock strength increases. Specifically, the IP method predicts better density and velocity profiles than the temperature profiles, which is mainly due to the nonequilibrium among the temperature components. DSMC simulations show that strong nonequilibrium exists across the shock wave. The temperature component normal to the shock wave, $T_{y}$, increases monotonically across the shock wave whereas the temperature component parallel to the shock wave, $T_{x}$, overshoots the overall temperature (by more than $20 \%$ for the Mach 5 case) in some part of the shock wave. More seriously, the normal component always lags behind the parallel component. However, in the current version of the IP method, only the overall temperature is preserved. Simulations show that the IP temperature follows the normal component of the temperature near the upstream and approaches the overall temperature downstream of the shock wave. This one-temperature version of the IP method is not able to capture fully the physical temperature profile, which also indicates that the IP method may require preserving several temperature components for problems where strong nonequilibrium exists among the temperature components.

One parameter characterizing a shock wave is the reciprocal shock thickness, which is a measure of the maximum density gradient. A plot of the reciprocal shock thickness is shown in Fig. 5 for a Mach number ranging from 1.2 to 10 . The data shown include results from experimental measurement, ${ }^{16}$ Navier-Stokes simulation, ${ }^{15}$ DSMC simulation, and IP simulation. There is some difference between the IP results and the experimental data, but this difference is much less than the difference between the Navier-Stokes results and the experimental

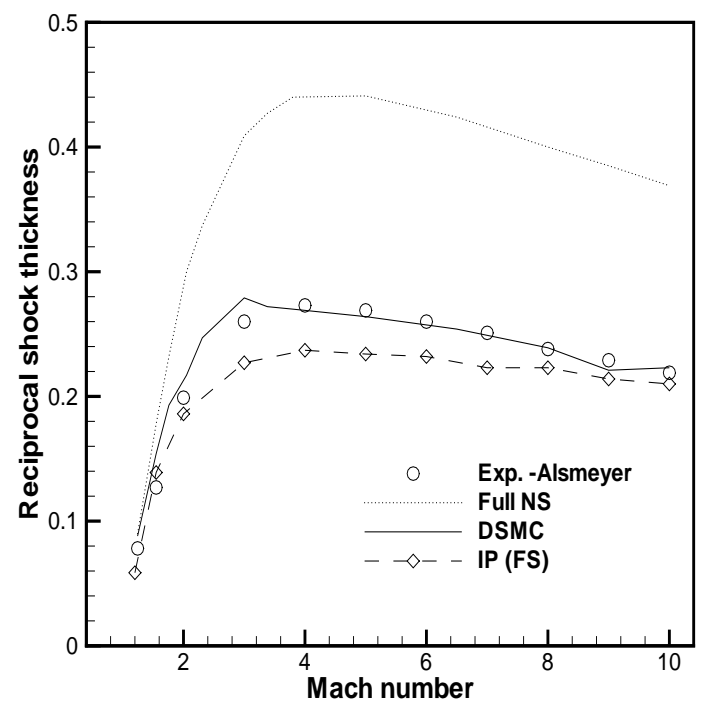

Figure 5. Comparison for the reciprocal shock data.

\section{B. Thermal Couette Flow}

Strong nonequilibrium also exists in other rarefied gas flows. The thermal Couette flow, where the rarefied effects can be easily characterized by the Knudsen (Kn) number, is another good example for evaluating the FS approach. The thermal Couette flow has been successfully simulated using the IP method when the additional 
energy flux model is employed. ${ }^{10}$ The purpose of revisiting this problem is to further evaluate the performance of the flux splitting version of the IP method.

The thermal Couette flow is a heat transfer flow between two parallel plates at different temperatures. In the present investigation, the two plates are $1 \mathrm{~m}$ apart, with one at $173 \mathrm{~K}$ and the other at $373 \mathrm{~K}$. Between the plates is an argon gas whose density is selected such that the Knudsen number of the flows at $273 \mathrm{~K}$ ranges from 0.01 to 100 . It is assumed that the thermal accommodation coefficient is 1.0 for both plates.

The temperature distribution of the flow is plotted in Fig. 6 where both DSMC and IP results are presented. The plot shows that the temperature jump at the plate surface increases and the temperature gradient decreases when the Knudsen number increases. Clearly, excellent agreement is obtained between the IP and DSMC results at all Knudsen numbers considered. Further analysis (Fig. 6(b)) shows that nonequilibrium among different temperature components also exists in this flow, but the discrepancy between different temperature components has no serious effects on the temperature distribution predicted by the IP method.

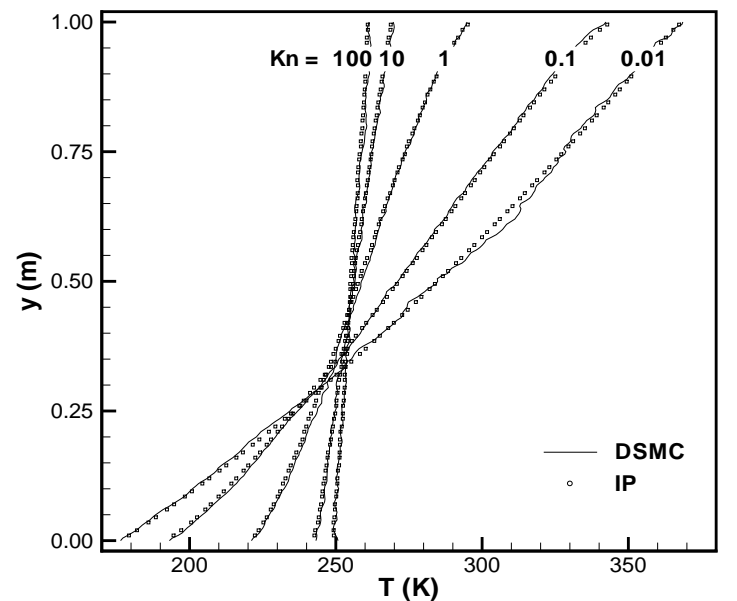

(a)

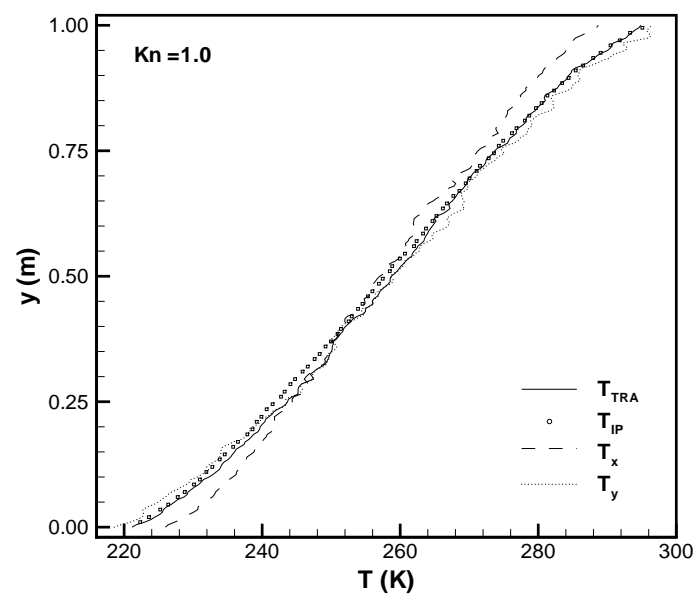

(b)

Figure 6. Temperature Distributions of Thermal Couette Flow. (a) temperature distribution at different Knudsen number, (b)Comparison of different temperature components.

\section{Discussion and Conclusion}

The information preservation (IP) method is a variant of the direct simulation Monte Carlo (DSMC) method, which is designed to reduce the statistical scatter of particle methods. In the IP method, simulated particles not only have the regular microscopic information as in the DSMC method but also preserve information at the macroscopic level. The IP method can dramatically reduce the statistical scatter by sampling the flow field using the preserved information instead of the microscopic information. The key part of the IP method is how to update the preserved information.

In this paper, we derived general expressions for the evolution of the preserved information. The main assumption used was that the governing equation for individual particle information followed the equation of change that governed the average information. In general, particles are assigned the preserved information at the macroscopic level when they are introduced into the computational domain. The preserved information is then carried by the particles and is adjusted during collisions. The preserved information should also be updated to include other effects, which is derived as the correlation term. Schemes for evaluating the correlation terms represent versions of the IP method. Two schemes were proposed in this paper. Namely, the local thermodynamic equilibrium approach and the flux splitting approach. It is shown that the previous additional energy flux model was a special case of the flux splitting approach.

Both the local thermodynamic equilibrium and flux splitting approaches are tested using numerical examples. It is found that the latter is a better approach. The flux splitting approach can capture the shock structure although there are slight discrepancies as compared with DSMC results when the shock strength increases. This approach is also able to predict the temperature distribution of the thermal Couette flow over the entire Knudsen number regime. Both flow examples show that nonequilibrium exists among temperature components. It is therefore believed that temperature components should be preserved in particles as information to better capture the flow physics of strongly nonequilibrium rarefied flows. 


\section{Acknowledgments}

This work was supported by the Air Force Office of Scientific Research under grant FA9550-05-1-0115. The authors would like to thank Dr. Wen-Lan Wang for helpful discussions. The views and conclusions contained herein are those of the authors and should not be interpreted as necessary representing the official policies or endorsements, either expressed or implied, of the AFOSR or the U.S. Government.

\section{References}

${ }^{1}$ Bird, G. A., Molecular Gas Dynamics and the Direct Simulation of Gas Flows, Clarendon Press, Oxford, 1994.

${ }^{2}$ Beskok, A., "Physical Challenges and Simulation of Micro Fluidic Transport," AIAA 2001-0718, 2001.

${ }^{3}$ Kaplan, C. R., and Oran, E. S., "Nonlinear Filtering for Low-Velocity Gaseous Microflows," AIAA Journal, Vol. 40, 2002, pp. 82-90.

${ }^{4}$ Pan, L. S., Liu, G. R., Khoo, B. C., and Song, B., "Modified Direct Simulation Monte Carlo Method for Low-Speed Microflows," Journal of Micromechanics and Microengineering, Vol. 10, 2001, pp. 21-27.

${ }^{5}$ Pan, L. S., Ng, T. Y., Xu, D., and Lam, K. Y., "Molecular Block Model Direct Simulation Monte Carlo Method for Low Velocity Microgas Flows," Journal of Micromechanics and Microengineering, Vol. 11, 2002, pp. 181-188.

${ }^{6}$ Fan, J., and Shen, C., "Statistical Simulation of Low-Speed Rarefied Gas Flows," Journal of Computational Physics, Vol. 167, 2001, pp. 393-412.

${ }^{7}$ Sun Q., "Information Preservation Methods for Modeling Micro-Scale Gas Flows," Ph.D. Dissertation, Dept. of Aerospace Engineering, Univ. of Michigan, Ann Arbor, MI, 2003.

${ }^{8}$ Fan, J., and Shen, C., "Statistical Simulation of Low-Speed Unidirectional Flows in Transition Regime," Proceedings of the $21^{\text {st }}$ International Symposium on Rarefied Gas Dynamics, edited by R. Brun, et al., Marseille, 1999, pp. 245-252.

${ }^{9}$ Cai, C., Boyd, I. D., Fan, J., and Candler, G., "Direct Simulation Methods for Low-Speed Microchannel Flows," Journal of Thermophysics and Heat Transfer, Vol. 14, 2000, pp. 368-378.

${ }^{10}$ Sun, Q., and Boyd, I. D., "A Direct Simulation Method for Subsonic, Micro-Scale Gas Flows," Journal of Computational Physics, Vol. 179, 2002, pp. 400-425.

${ }^{11}$ Sun, Q., Boyd, I. D., and Candler, G. V., "Numerical Simulation of Gas Flow over Micro-Scale Airfoils," Journal of Thermophysics and Heat Transfer, Vol. 16, 2001, pp. 171-179.

${ }^{12}$ Shen, C., Fan, J., and Xie, C., "Statistical Simulation of Rarefied Gas Flows in Microchannels," Journal of Computational Physics, Vol. 189, 2003, pp. 512-526.

${ }^{13}$ Sun, Q., and Boyd, I. D., "Drag on a Flat Plate of Low Reynolds Number Gas Flows," AIAA Journal, Vol. 42, 2004, pp. 1066-1072.

${ }^{14}$ Sun, Q., and Boyd, I. D., "Flat-Plate Aerodynamics of Very Low Reynolds Number," Journal of Fluid Mechanics, Vol. 502, 2004, pp. 199-206.

${ }^{15}$ Wang, W-L, and Boyd, I.D., "A New Energy Flux Model in the DSMC-IP Method for Nonequilibrium Flows," AIAA 2003-3774, 2003.

${ }^{16}$ Alsmeyer, H., "Density Profiles in Argon and Nitrogen Shock Waves Measured by the Absorption of an Electron Beam," Journal of Fluid Mechanics, Vol. 74, 1976, pp. 497-523. 